\title{
Flora de Inselbergues do Monumento Natural Monólitos de Quixadá, no sertão central do Ceará
}

\author{
Regis da Cruz Paulino', Vaneicia dos Santos Gomes' \& Andréa Pereira Silveira²
}

\author{
' Universidade Estadual do Ceará, Faculdade de Educação, Ciências e Letras do Sertão Central, CEP 63. 9000-000, Quixadá, CE, Brasil. \\ regiscruz24@gmail.com; vaneicia.gomes@uece.br \\ ${ }^{2}$ Universidade Estadual do Ceará, Faculdade de Educação de Itapipoca, CEP 60.500-000, Itapipoca, CE, Brasil. andrea.silveira@uece.br
}

Recebido em 01.V.2016

Aceito em 30.VII.2018

DOI 10.21826/2446-8231201873110

RESUMO - Inselbergues são ilhas rochosas que ocorrem em montes isolados ou aglomerados, como o Monumento Natural Monólitos de Quixadá no semiárido Cearense. Visamos conhecer a composição florística destes inselbergs, as síndromes de dispersão, as formas de crescimento e de vida predominantes e como a diversidade de micro-habitats influencia esses atributos. Para esse estudo realizamos coletas mensais da flora vascular durante dois anos em dois inselbergues Quixadaenses. Reconhecemos os micro-habitats cacimba, canal de drenagem, fenda, fissura, paredão rochoso, depressão rasa, depressão profunda, cinturão florestado e caverna. Registramos 107 espécies em 45 famílias, sendo Fabaceae (13 espécies), Apocynaceae (11), Euphorbiaceae (06), Bromeliaceae (05) e Cactaceae (04) as mais representativas. A riqueza e composição florística diferiram de outros inselbergues. Predominaram espécies zoocóricas (43), fanerófitas (64) e árvores (45), contrário ao esperado para regiões semiáridas. Concluímos que estas diferenças se devem às características intrínsecas dos nove micro-habitats reconhecidos, como quantidade de substrato e disponibilidade hídrica.

Palavras-chave: afloramento rochoso, caatinga, forma de vida, micro-habitat

ABSTRACT - Flora of the inselbergs of the Natural Monument Monólitos de Quixada in the central sertão of Ceará. Inselbergs are rocky outcrop islands that occur in isolated mountains and villages, such as the Natural Monument of Monólitos de Quixadá in semiarid Ceará. We aims to know the floristic comparison of these inselbergs, the patterns of dispersal, and the predominant growth forms and life; as well as how the diversity of the microhabitat influences these attributes. For this study we carried out monthly collections of the vascular flora of two inselbergs of the Natural Monument Monólitos de Quixada for two years. We recognized the microhabitats: rock pools, drainage channel, crevices, clefts, rock wall, shallow depression, deep depression, forested belt and cavern. We recorded 107 species in 45 families, being that Fabaceae (13 species), Apocynaceae (11), Euphorbiaceae (06), Bromeliaceae (05) and Cactaceae (04) were most representative. Their richness and composition differs from other inselbergs. Zoochoric species (43), phanerophytes (64) and trees (45) were predominant, contrary to what was expected for semi-arid regions. We concluded that these differences are due to the intrinsic characteristics of the nine recognized microhabitats, such as amount of substrate and water availability.

Keywords: rocky outcrop, thorny savannah, life-forms, micro-habitat

\section{INTRODUÇÃO}

O termo inselbergue foi introduzido pelo geólogo alemão Bornhardt para se referir às rochas monolíticas ou grupos de rochas que se erguem abruptamente das planícies circundantes e, junto ao pediplano são as formações geológicas que mais caracterizam as regiões semiáridas (Ribeiro et al. 2010). Os inselbergues estão difundidos nos antigos escudos cristalinos continentais sendo particularmente frequentes nos trópicos e subtrópicos, mas também podem ser encontradas em zonas temperadas como o sudeste dos Estados Unidos e da Austrália (Barthlott et al. 1993, Porembski \& Barthlott 2000). Estes afloramentos rochosos são de granito e gnaisse, exceto os "tepuis" encontrados na Venezuela, que são compostos por quartzito e arenito (Gröger \& Huber 2007) e as "cangas" que são afloramentos ferruginosos predominantes em Minas Gerais (Carmo \& Jacobi 2013). No Brasil as formações graníticas são amplamente distribuídas no escudo cristalino, ao longo da costa tropical do Atlântico e são especialmente comuns na região nordeste (França et al. 1997).

Estes afloramentos rochosos constituem ambientes ecologicamente isolados, apresentando uma composição vegetacional com alta riqueza e endemismos de espécies (Porembski et al. 1997, Porembski et al. 1998, Parmentier et al. 2005). Estudos indicam que a diversidade de microhabitats associados às condições microclimáticas e geológicas são responsáveis pela diversidade de plantas nos inselbergues (Gröger \& Huber 2007). Por serem áreas de vegetação isolada eles constituem verdadeiras ilhas de vegetação natural e podem ser consideradas como refúgios ecológicos para muitas espécies de plantas nativas (Lumaret et al. 1997) e podem funcionar de maneira análoga às ilhas oceânicas, que são utilizadas para o desenvolvimento de diversos modelos biogeográficos (MacArthur \& Wilson 1967, Lumaret et al. 1997), permitindo testar a relação entre a riqueza de espécies e os mecanismos de adaptação associados ao isolamento. Segundo Porembski et al. 
(1997), as plantas que colonizam áreas isoladas do tipo inselbergues apresentam adaptações específicas à falta de água e a escassez de substrato, com composição florística e distribuição espacial de espécies bastante heterogêneas devido aos vários tipos de micro-habitats disponíveis.

Outra característica peculiar dos inselbergues "lato sensu" é que eles podem variar de tamanho, desde aqueles com menos de $100 \mathrm{~m}$ de altura até aqueles que formam maciços montanhosos chegando a mais de $1000 \mathrm{~km}$ de diâmetro (Porembski 2007) e $3000 \mathrm{~m}$ de altura (Gröger \& Huber 2007). O grau de isolamento geográfico também é variado, podendo ocorrer em montes isolados por centenas de quilômetros ou formar aglomerados densos com afloramentos ocorrendo em distâncias de apenas alguns quilômetros (Porembski 2007). Este é o caso do município de Quixadá no estado do Ceará que possui um polígono de tombamento composto por 13 inselbergues em uma extensão de $20 \mathrm{~km}$ (WFMA 2015). Estes inselbergues podem se assemelhar a figuras antropomórficas ou animalescas, e são regionalmente conhecidos como monólitos ou simplesmente pedras, acrescida de um epíteto especifico de acordo com a característica geomorfológica mais evidente da rocha, a exemplo da Pedra da Galinha Choca.

As pesquisas sobre vegetação em afloramentos rochosos realizadas no Brasil podem ser divididas de acordo com Silva (2016) em dois períodos. No primeiro período, entre 1980-2007 os estudos se concentraram na região Sudeste principalmente na Mata Atlântica e no Cerrado com alguns trabalhos de destaque como os de Meirelles et al. (1999), Porembski et al. (1998), Martinelli \& Forzza (2006), e Medina et al. (2006). No segundo período entre 2008-2016, as publicações se concentraram na Caatinga, Mata Atlântica e Cerrado (Silva 2016). Especificamente no nordeste brasileiro, foram realizados estudos com a flora de inselbergues em Pernambuco por Martins \& Alves (2008) e Gomes \& Alves (2009); na Bahia por França et al. (1997) e Moraes et al. (2009); na Paraíba por Almeida et al. (2008) e Porto et al. (2008) e no Ceará com um único estudo realizado por Araújo et al. (2008). Embora tenha crescido o número de trabalhos nos inselbergues nos últimos anos, o conhecimento sobre a flora dos inselbergues é insuficiente, especialmente, em relação à conservação (Silva 2016).

Levando-se em consideração a distribuição peculiar dos inselbergues que compõem o Monumento Natural Monólitos de Quixadá, as pressões antrópicas geradas nesses ecossistemas e a necessidade de conhecimento e conservação dessas formações, procuramos com este trabalho responder as seguintes questões: qual a composição florística dos micro-habitats, quais as síndromes de dispersão, quais as formas de crescimento e as formas de vida predominantes em dois inselbergues Quixadaenses? E ainda, a composição florística destes inselbergues é influenciada pela diversidade de micro-habitats? Como?

Esperamos registrar nos inselbergues Quixadaenses elevada representatividade de espécies, predomínio da forma de crescimento ervas anuais, da forma de vida terófito e da síndrome de dispersão anemocórica. Se isto ocorrer, podemos concluir que estes atributos estão refletindo a aridez climática dessas ilhas rochosas semelhante ao entorno (ver Araújo et al. 2008). Do contrário, condições microclimáticas locais diferentes devido a ocorrência de micro-habitats distintos, podem ser responsáveis pelas alterações nos padrões já documentados em outros inselbergues com latitudes coincidentes (França et al. 2005) e explicar as diferenças nos parâmetros aqui investigados.

\section{MATERIAL E MÉTODOS}

\section{Área de Estudo}

O município de Quixadá, localiza-se no sertão central do estado do Ceará, dista $160 \mathrm{~km}$ da capital Fortaleza e possui clima tropical quente semiárido com inverno seco e verão chuvoso - BSh de Köppen-Geiger. A pluviosidade média anual é de $731 \mathrm{~mm}$, concentrada nos meses de janeiro a junho, considerando a média histórica de 1974-2014 (FUNCEME 2015). A temperatura média anual é de $28,2^{\circ} \mathrm{C}$, a média das máximas é $33,4^{\circ} \mathrm{C}$ e a média das mínimas é de $22,9^{\circ} \mathrm{C}$ (INMET 2015).

Nessa região está situado o "Monumento Natural Monólitos de Quixadá", que compreende uma Unidade de Conservação de Proteção Integral (entre 04 $54^{\circ}$ 'S $05^{\circ} 02^{\prime} \mathrm{S}$ e $38^{\circ} 53^{\prime} \mathrm{W}-39^{\circ} 06^{\prime} \mathrm{W}$, com $16.635,59 \mathrm{ha}$ ), criada pelo Decreto Estadual No 26.805 de 25 de outubro de 2002, para proteger um conjunto de inselbergues, regionalmente denominados de monólitos ou serrotes (Menezes et al. 2010). Em 2004, a área dos monólitos foi tombada pelo Instituto de Patrimônio Histórico e Artístico Nacional (IPHAN) e desde 2010 faz parte da Associação Mundial de Montanhas Famosas (WFMA 2015). Os inselbergues de Quixadá são circundados pela vegetação de Savana Estépica (Caatinga) inventariada por Costa et al. 2007.

\section{Seleção dos inselbergues e identificação dos micro- habitats}

A pesquisa foi desenvolvida em dois inselbergues Quixadaenses conhecidos como Pedra Derretida e Pedra do Padre. Estes inselbergues apresentam superfície heterogênea com muitas fissuras e faces diferenciadas em relação à inclinação, grau de isolamento e estrutura da superfície, o que representa uma grande variedade de microhabitats. Para a caracterização dos micro-habitats, adotamos a classificação proposta por Barthlott et al. (1993) e Ibisch et al. (1995), com as devidas atualizações de Porembski (2007): Fendas; Fissuras; Cacimbas; Paredão Rochoso; Canal de Drenagem; Cinturão Florestado e Depressão na rocha - que foi subdividido em Depressão Rasa e Depressão Profunda; e adicionamos o termo Caverna, para fazer referencia aos vegetais presentes nas áreas constantemente sombreadas pela rocha.

\section{Caracterização da flora, formas de crescimento, formas de vida e síndromes de dispersão}

Realizamos coletas aleatórias no período de julho de 2009 a julho de 2011, com posterior prensagem e 
herborização dos espécimes férteis da flora vascular. As exsicatas foram depositadas no herbário da Faculdade de Educação, Ciências e Letras do Sertão Central da Universidade Estadual do Ceará (FECLESC-UECE). Fizemos também comparação do material coletado com aquele pertencente aos acervos do Herbário Rodolfo Teófilo (HERT) da Universidade Estadual do Ceará e do Herbário Prisco Bezerra (EAC) da Universidade Federal do Ceará.

O levantamento da flora se deu desde a base do afloramento rochoso até o topo, percorrendo todos os microhabitats. A área da base até $5 \mathrm{~m}$ afastada do inselbergue, também foi abrangida, segundo a metodologia empregada por França et al. (1997). Adotamos a classificação Angiosperm Phylogeny Group (APG IV 2016) e a conferência da grafia dos nomes científicos e dos autores foi realizada através dos sites International Plant Names Index (IPNI 2015) e Lista de Espécies da Flora e Fungos do Brasil (JBRJ 2016).

As formas de vida, as formas de crescimento e as síndromes de dispersão foram determinadas no momento da coleta ou posteriormente, com auxílio de literaturas especializadas. Adotamos o sistema de Raunkiaer (1934) para classificar as formas de vida, o qual considera a posição das partes regenerativas dos vegetais como adaptações às condições ambientais, enquadrando as espécies em: fanerófito (fan); caméfito (cam); hemicriptófito (hem); geófito (geo) e terófito (ter). Para a determinação das formas de crescimento utilizamos os trabalhos de Araújo et al. (2005) classificando as plantas em: árvores (arv); arbustos (arb); subarbustos (sub); ervas (erv); trepadeiras lenhosas (trl) e trepadeiras herbáceas (trh). E para determinar o modo de dispersão dos diásporos utilizamos a classificação de Van der Pijl (1969), onde são analisadas as adaptações morfológicas dos frutos e também consultamos os trabalhos de Griz \& Machado (2001) categorizando as espécies em: autocórica (aut); anemocórica (ane) e zoocórica (zoo).

\section{RESULTADOS E DISCUSSÃO}

\section{Caracterização Florística}

Registramos 107 espécies pertencentes a 45 famílias (Quadro 1), confirmando a alta riqueza de espécies esperada para ambientes isolados como os inselbergues (Ratter et al. 1996, Parmentier et al. 2005, Porembski 2007). Registramos também um elevado número de gêneros compostos por apenas uma espécie o que também está de acordo com o padrão registrado para ilhas rochosas (França et al. 1997), pois esses afloramentos funcionam como importantes centros de origem e diversidade de plantas que toleram ou evitam à dessecação (Porembski \& Barthlott 2000, Burke 2004).

As famílias mais representativas foram: Fabaceae (13 espécies.), Apocynaceae (11), Euphorbiaceae (06), Bromeliaceae (05), Cactaceae, Malvaceae e Araceae com 04 espécies cada, e somaram juntas $44 \%$ das espécies inventariadas (Quadro 1). Os gêneros com maior número de espécies foram Tillandsia (04 espécies), Mimosa (03),
Croton, Pilosocereus, Commelina, Passiflora, Portulaca e Solanum, com duas espécies cada. Estes resultados divergem do levantamento florístico realizado por Araújo et al. (2008) em outro inselbergue Quixadaense, uma vez que os autores documentaram 77 espécies, sendo Fabaceae (11 espécies), Poaceae (10), Euphorbiaceae (05), Asteraceae (04) e Convolvulaceae (04) as famílias mais ricas. Além disso, os gêneros Campomanesia, Cyperus, Digitaria, Evolvulus e Paspalum registrados no estudo de Araújo et al. (2008), não foram encontrados neste estudo. Essa diferenciação na composição florística entre inselbergues próximos foram documentados na África por Parmentier (2003), ao registrar que formações rochosas próximas umas a outras $(100$ a $500 \mathrm{~m})$ apresentaram uma vegetação similar, porém com algum grau de diferenciação. Porembski (2007) e Scarano (2007) explicaram essa diferenciação florística como sendo resultado das características microclimáticas e pedológicas peculiares (estresse hídrico, insolação e temperaturas elevadas) que sustentam uma vegetação altamente especializada.

Em relação aos inselbergues situados em regiões com diferenças climáticas marcantes, o padrão documentado é o de que Poaceae é uma das famílias com maior número de espécies em inselbergues situados em regiões de clima seco (Ibisch et al. 1995, Araújo et al. 2008, Porto et al. 2008), mas em climas mais úmidos, Bromeliaceae e Orchidaceae estão entre as famílias mais numerosas em número de espécies (França et al. 2005, Martinelli \& Forzza 2006, Caiafa \& Silva 2007, Scarano 2007, Sarthou et al. 2009). Contrário a este padrão, nós registramos nos dois inselbergues aqui estudados uma grande representatividade de Bromeliaceae (05 espécies), enquanto Poaceae foi representada por apenas uma espécie. Este resultado também demonstra que diferenças florísticas podem ocorrer em escalas regionais e locais. Assim, atribuímos as diferenças de composição e número de espécies (107 espécies neste estudo e 77 no levantamento de Araújo et al. 2008) a uma maior diversidade de sítios de estabelecimento nos inselbergues Pedra Derretida e Pedra do Padre, os quais apresentaram nove micro-habitat em comparação aos três micro-habitat (depressão, fissura e rocha exposta) descritos no inselbergue inventariado por Araújo et al. (2008), ambos situados no município de Quixadá, nordeste semiárido do Brasil. Esta explicação é sustentada pelas premissas de que diferentes modos de escultura da superfície da rocha produzem uma grande variedade e tamanhos diferentes de depressões, barrancos, canais e rachaduras; cada uma com diferenças nas quantidades e qualidade do solo e no abastecimento de água, criando uma variedade de sítios de estabelecimento, responsáveis pela ocorrência das mais diversas espécies vegetais (Barthlott et al. 1993, Porembski \& Barthlott 2000, Burke 2002, Parmentier et al. 2005, Gröger \& Huber 2007).

Os nove micro-habitat descritos neste estudo não encerram uma padronização de conceitos, mas sim, uma adequação das classificações já propostas à realidade dos inselbergues amostrados. Neste contexto segue a descrição 
Quadro 1. Flora vascular de Inselbergues de Quixadá-Ceará, com a respectiva Forma de Crescimento (FC): árvore (arv), arbusto (arb), subarbusto (sub), erva (erv), trepadeira herbácea (trh), trepadeira lenhosa (trl); Forma de Vida (FV): terófito (ter), fanerófito (fan), caméfito (cam), geófito (geo), hemicriptófito (hem); Micro-habitat (MI): cacimba (cac), canal de drenagem (cd), caverna (cav), fenda (fen), fissura (fis), paredão rochoso (pr), depressão rasa (dr), depressão profunda (dp), cinturão florestado (cf) e Síndrome de Dispersão (SD): anemocórica (ane), zoocórica (zoo), autocórica (aut).

\begin{tabular}{|c|c|c|c|c|}
\hline Família/Espécie & $\mathrm{FC}$ & FV & MI & $\mathrm{SD}$ \\
\hline \multicolumn{5}{|l|}{ Acanthaceae } \\
\hline Ruellia sp. & sub & ter & $\mathrm{dp}$ & ane \\
\hline R. asperula (Mart. \& Nees) Lindau & sub & ter & $\mathrm{dp}$ & ane \\
\hline \multicolumn{5}{|l|}{ Alismataceae } \\
\hline Echinodorus lanceolatus Rataj & erv & hem & $\mathrm{cac}$ & zoo \\
\hline \multicolumn{5}{|l|}{ Amaryllidaceae } \\
\hline Habranthus itaobinus Ravenna & erv & geo & $\mathrm{dr}$ & ane \\
\hline \multicolumn{5}{|l|}{ Anacardiaceae } \\
\hline Litraea sp. & $\operatorname{arv}$ & fan & cf, fis & aut \\
\hline \multicolumn{5}{|l|}{ Apocynaceae } \\
\hline Aspidosperma pyrifolium Mart. & $\operatorname{arv}$ & fan & $\mathrm{dp}$ & ane \\
\hline Calotropis procera (Aiton) W.T. Aiton & sub & fan & $\mathrm{dp}, \mathrm{cd}$ & ane \\
\hline Cryptostegia madagascariensis Bojer & $\operatorname{trl}$ & fan & $\mathrm{dp}$, fis & ane \\
\hline Ditassa sp. & erv & cam & fen, $\mathrm{dr}$ & ane \\
\hline Mandevilla sp. & $\operatorname{trh}$ & geo & $\mathrm{dp}$ & ane \\
\hline M. tenuifolia (J.C. Mikan) Woodson & $\operatorname{trh}$ & geo & $\mathrm{dr}$ & ane \\
\hline Marsdenia altissima (Jacq.) Dugand & $\operatorname{trh}$ & fan & $\mathrm{dr}$ & ane \\
\hline M. megalantha Goyder \& Morillo & $\operatorname{trh}$ & fan & $\mathrm{dr}$ & ane \\
\hline Peplonia sp. & erv & ter & $\mathrm{dr}$ & ane \\
\hline Petalostelma martianum (Decne.) E. Fourn. & $\operatorname{trh}$ & fan & fis & ane \\
\hline Rauvolfia ligustrina Willd. & arb & fan & $\mathrm{dp}$ & ane \\
\hline \multicolumn{5}{|l|}{ Aquifoliaceae } \\
\hline Ilex sp. 1 & $\operatorname{arv}$ & fan & cav & zoo \\
\hline Ilex sp. 2 & $\operatorname{arv}$ & fan & fis & zoo \\
\hline \multicolumn{5}{|l|}{ Araceae } \\
\hline Lemna minuta Kunth & erv & ter & $\mathrm{cac}$ & aut \\
\hline Philodendron acutatum Schott & erv & hem & cav, fis & zoo \\
\hline Spathicarpa sagittifolia Schott & erv & geo & cav & zoo \\
\hline Taccarum peregrinum (Schott) Engl. & erv & geo & cav, $\mathrm{dr}$ & zoo \\
\hline \multicolumn{5}{|l|}{ Arecaceae } \\
\hline Copernicia prunifera (Mill.) H.E. Moore & $\operatorname{arv}$ & fan & $\mathrm{dp}$ & zoo \\
\hline \multicolumn{5}{|l|}{ Bixaceae } \\
\hline Cochlospermum vitifolium (Willd.) Spreng. & $\operatorname{arv}$ & fan & $\mathrm{dp}$ & ane \\
\hline \multicolumn{5}{|l|}{ Boraginaceae } \\
\hline Cordia insignis Cham. & $\operatorname{arv}$ & fan & fis & zoo \\
\hline C. oncocalyx Allemão & $\operatorname{arv}$ & fan & $\mathrm{cf}$ & zoo \\
\hline Tournefortia salzmannii DC. & arb & fan & $\mathrm{dp}$ & zoo \\
\hline \multicolumn{5}{|l|}{ Bromeliaceae } \\
\hline Encholirium spectabile Mart. ex Schult. \& Schult.f. & erv & cam & pr, dr & ane \\
\hline Tillandsia recurvata (L.) L. & erv & cam & $\mathrm{dp}$ & ane \\
\hline Tillandsia sp. 1 & erv & cam & $\mathrm{pr}$ & ane \\
\hline Tillandsia sp. 2 & erv & cam & pr & ane \\
\hline Tillandsia sp. 3 & erv & cam & $\mathrm{dp}$ & ane \\
\hline \multicolumn{5}{|l|}{ Cactaceae } \\
\hline Cereus jamacaru DC. & $\operatorname{arv}$ & fan & fis, cd & zoo \\
\hline Pilosocereus gounellei (F.A.C. Weber) Byles \& Rowley & $\operatorname{arv}$ & fan & fis, dr & zoo \\
\hline P. chrysostele (Vaupel) Byles \& G.D. Rowley & $\operatorname{arv}$ & fan & fis, dr & zoo \\
\hline Tacinga inamoena (K. Schum.) N.P. Taylor \& Stuppy & arb & fan & fis, dr & zoo \\
\hline \multicolumn{5}{|l|}{ Capparaceae } \\
\hline Capparis flexuosa (L.) L. & $\operatorname{arv}$ & fan & $\mathrm{dp}$, fis & zoo \\
\hline Crateva trapia $L$. & arv & fan & $\mathrm{cf}$ & zoo \\
\hline \multicolumn{5}{|l|}{ Combretaceae } \\
\hline Combretum leprosum Mart. & arb & fan & $\mathrm{dp}$ & ane \\
\hline \multicolumn{5}{|l|}{ Commelinaceae } \\
\hline Commelina benghalensis L. & erv & ter & $\mathrm{cd}, \mathrm{dr}, \mathrm{dp}$ & aut \\
\hline
\end{tabular}


Quadro 1. Cont.

\begin{tabular}{|c|c|c|c|c|}
\hline Família/Espécie & $\mathrm{FC}$ & FV & MI & SD \\
\hline C. erecta $\mathrm{L}$. & erv & ter & $\mathrm{cd}, \mathrm{dr}, \mathrm{dp}$ & aut \\
\hline Tradescantia ambigua Mart. & erv & ter & $\mathrm{dr}$ & aut \\
\hline \multicolumn{5}{|l|}{ Cannabaceae } \\
\hline Trema micrantha (L.) Blume & $\operatorname{arv}$ & fan & fis, dp, cf & aut \\
\hline \multicolumn{5}{|l|}{ Convolvulaceae } \\
\hline Morfoespécie 1 & $\operatorname{trh}$ & ter & $\mathrm{dr}$ & aut \\
\hline \multicolumn{5}{|l|}{ Ebenaceae } \\
\hline Diospyros hispida A. DC. & arv & fan & fis & zoo \\
\hline Diospyros sp. & arv & fan & fis & zoo \\
\hline \multicolumn{5}{|l|}{ Euphorbiaceae } \\
\hline Cnidoscolus urens (L.) Arthur & arb & fan & $\mathrm{dp}$ & aut \\
\hline Croton moritibensis Baill. & arb & fan & $\mathrm{dp}$, fis, cd & aut \\
\hline C. sonderianus Müll. Arg. & arb & fan & $\mathrm{dp}$, fis, cd & aut \\
\hline Dalechampia brasiliensis Lam. & $\operatorname{trh}$ & ter & $\mathrm{dr}$ & aut \\
\hline Jatropha mollissima (Pohl) Baill. & arb & fan & $\mathrm{dp}$ & aut \\
\hline Manihot glaziovii Müll. Arg. & $\operatorname{arv}$ & fan & $\mathrm{dp}$ & aut \\
\hline \multicolumn{5}{|l|}{ Fabaceae } \\
\hline Aeschynomene matosii Afr. Fern. & erv & ter & $\mathrm{dr}$ & aut \\
\hline Anadenanthera colubrina (Vell.) Brenan & arv & fan & cf, fis & aut \\
\hline Bauhinia pentandra (Bong.) D.Dietr. & $\operatorname{arv}$ & fan & fis, dp & aut \\
\hline Centrosema pascuorum Mart. ex Benth. & $\operatorname{trh}$ & ter & $\mathrm{dr}$ & aut \\
\hline Chloroleucon dumosum (Benth.) G.P. Lewis & arv & fan & fis & aut \\
\hline Hymenaea courbaril L. & $\operatorname{arv}$ & fan & fis & zoo \\
\hline Luetzelburgia auriculata (Allemão) Ducke & $\operatorname{arv}$ & fan & fis & ane \\
\hline Mimosa caesalpiniifolia Benth. & arv & fan & fis, cf & aut \\
\hline M. calumbi (Willd.) Poir. & arv & fan & fis & aut \\
\hline M. tenuifolia L. & arv & fan & $\mathrm{dp}$, fis & aut \\
\hline Piptadenia stipulacea (Benth.) Ducke & $\operatorname{arv}$ & fan & cf, fis & aut \\
\hline Stylosanthes humilis Kunth & erv & ter & $\mathrm{dr}$ & aut \\
\hline Zornia sericea Moric. & erv & ter & $\mathrm{dr}$ & aut \\
\hline \multicolumn{5}{|l|}{ Gentianaceae } \\
\hline Schultesia guianensis (Aubl.) Malme & erv & ter & $\mathrm{dr}$ & aut \\
\hline \multicolumn{5}{|l|}{ Malpighiaceae } \\
\hline Morfoespécie 2 & arv & fan & fis & zoo \\
\hline \multicolumn{5}{|l|}{ Marantaceae } \\
\hline Calathea sp. & erv & ter & fis & zoo \\
\hline \multicolumn{5}{|l|}{ Malvaceae } \\
\hline Pseudobombax marginatum (A.St.-Hil.) A. Robyns & $\operatorname{arv}$ & fan & fis & ane \\
\hline Helicteres mollis C. Presl & $\operatorname{arv}$ & fan & fis, cf, dp & aut \\
\hline Herissantia crispa (L.) Brizicky & erv & ter & $\mathrm{dr}$ & ane \\
\hline Pavonia sp. & erv & ter & $\mathrm{dr}$ & ane \\
\hline \multicolumn{5}{|l|}{ Meliaceae } \\
\hline Cedrela odorata $\mathrm{L}$. & $\operatorname{arv}$ & fan & fis & ane \\
\hline C. fissilis Vell. & $\operatorname{arv}$ & fan & fis & ane \\
\hline \multicolumn{5}{|l|}{ Moraceae } \\
\hline Ficus christianii Carauta & arv & fan & fis, cf & zoo \\
\hline Maclura tinctoria (L.) D. Don ex Steud. & $\operatorname{arv}$ & fan & fis & zoo \\
\hline \multicolumn{5}{|l|}{ Myrtaceae } \\
\hline Morfoespécie 3 & arv & fan & fis & zoo \\
\hline Morfoespécie 4 & arv & fan & fis & zoo \\
\hline Morfoespécie 5 & $\operatorname{arv}$ & fan & fis & zoo \\
\hline Morfoespécie 6 & $\operatorname{arv}$ & fan & fis & zoo \\
\hline Morfoespécie 7 & $\operatorname{arv}$ & fan & fis & zoo \\
\hline Morfoespécie 8 & arv & fan & fis & zoo \\
\hline \multicolumn{5}{|l|}{ Olacaceae } \\
\hline Ximenia americana L. & arv & fan & fis & zoo \\
\hline \multicolumn{5}{|l|}{ Onagraceae } \\
\hline Ludwigia leptocarpa (Nutt.) H. Hara & erv & ter & $\mathrm{cac}$ & aut \\
\hline
\end{tabular}




\begin{tabular}{|c|c|c|c|c|}
\hline Família/Espécie & $\mathrm{FC}$ & FV & MI & $\mathrm{SD}$ \\
\hline \multicolumn{5}{|l|}{ Orquidaceae } \\
\hline Epidendrum sp. & erv & hem & $\mathrm{pr}$ & ane \\
\hline Cyrtopodium saintlegerianum Rchb. $\mathrm{f}$. & erv & hem & $\mathrm{dr}$ & ane \\
\hline \multicolumn{5}{|l|}{ Oxalidaceae } \\
\hline Oxalis triangularis A. St.-Hil. & erv & ter & $\mathrm{dr}$ & aut \\
\hline \multicolumn{5}{|l|}{ Passifloraceae } \\
\hline Passiflora cincinnata Mast. & $\operatorname{trl}$ & fan & fis & zoo \\
\hline P. foetida $\mathrm{L}$. & trh & ter & $\mathrm{dr}$ & zoo \\
\hline \multicolumn{5}{|l|}{ Phylantaceae } \\
\hline Phylantus sp. & arv & fan & fis & aut \\
\hline \multicolumn{5}{|l|}{ Poaceae } \\
\hline Melinis repens (Willd.) Zizka & erv & ter & fen & aut \\
\hline \multicolumn{5}{|l|}{ Portulacaceae } \\
\hline Portuluca sp. 1 & erv & ter & $\mathrm{dr}$ & aut \\
\hline Portuluca sp. 2 & erv & ter & $\mathrm{dr}$ & aut \\
\hline \multicolumn{5}{|l|}{ Pteridaceae } \\
\hline Adiantum deflectens Mart. & erv & hem & cav & aut \\
\hline Doryopteris concolor (Langsd. \& Fisch.) Kuhn & erv & hem & cav & aut \\
\hline \multicolumn{5}{|l|}{ Ramnaceae } \\
\hline Ziziphus joazeiro Mart. & arv & fan & fis & zoo \\
\hline \multicolumn{5}{|l|}{ Rubiaceae } \\
\hline Spermacoce sp. & erv & ter & $\mathrm{dr}$ & ane \\
\hline Randia sp. & arb & fan & $\mathrm{dp}$ & zoo \\
\hline \multicolumn{5}{|l|}{ Rutaceae } \\
\hline Sigmatanthus trifoliatus Huber ex Emmerich & arv & fan & fis & zoo \\
\hline \multicolumn{5}{|l|}{ Salicaceae } \\
\hline Banara sp. & arv & fan & fis & zoo \\
\hline Casearia sp. & arv & fan & fis, cf, dp & zoo \\
\hline \multicolumn{5}{|l|}{ Santalaceae } \\
\hline Phoradendron sp. & erv & cam & $\mathrm{cf}$ & zoo \\
\hline \multicolumn{5}{|l|}{ Sapindaceae } \\
\hline Cardiospermum corindum $\mathrm{L}$. & $\operatorname{trl}$ & fan & fis & zoo \\
\hline \multicolumn{5}{|l|}{ Solanaceae } \\
\hline Solanum sp. 1 & arb & fan & $\mathrm{dp}$ & zoo \\
\hline Solanum sp. 2 & arb & fan & $\mathrm{dp}$ & zoo \\
\hline \multicolumn{5}{|l|}{ Verbenaceae } \\
\hline Lantana camara $\mathrm{L}$. & erv & ter & $\mathrm{dr}$ & zoo \\
\hline \multicolumn{5}{|l|}{ Vitaceae } \\
\hline Cissus simsiana Schult. \& Schult. f. & $\operatorname{trl}$ & fan & fis & zoo \\
\hline
\end{tabular}

destes sítios, acompanhada dos termos em inglês utilizados por Porembski (2007) e com referência para as espécies mais representativas de cada um deles:

As Cacimbas - "rock pools" correspondem a microhabitat sazonalmente cheio de água que cobre uma ampla gama de tamanho, forma e profundidade. Apresentaram vegetação apenas enquanto acumularam água, e foram ocupadas na área de estudos pelos hidrófitos Ludwigia leptocarpa (Nutt.) H. Hara, Lemna minuta Kunth e Echinodorus lanceolatus Rataj.

As Fendas - “crevices” são superficiais, acumulam pouco substrato e nelas encontramos apenas as herbáceas terofíticas Melinis repens (Willd.) Zizka, e Ditassa sp., esta última em extrema adaptação vascular à rocha.

As Fissuras - "clefts" são mais profundas do que as fendas e são caracterizadas por reter mais substrato, oferecendo condições para o desenvolvimento de espécies perenes, o que explica o porquê de um grande número de fanerófitos (arbustos e árvores de pequeno porte) deste estudo ter sido registrada nesse micro-habitat, semelhante ao documentado em Araújo et al. (2008).

Os Paredões Rochosos - "rocky slopes" são caracterizados por rocha com inclinações próximas de $90^{\circ}$ - de onde os únicos colonizadores se restringiram aos indivíduos das diferentes espécies de Tillandsia e Epidendrum. Rochas expostas com inclinações menos acentuadas tiveram como principal componente Encholirium spectabile descrita nos trabalhos de França et al. (1997), como dominante em afloramentos rochosos do semiárido. Estas espécies ocorrem com grande número de indivíduos, preferencialmente nas áreas ensolaradas e abertas.

Os Canais de Drenagem - "drainage channels" são vias de escoamento de água que ocorrem na superfície da rocha e neste micro-habitat foram registradas ervas e 
arbustos como Commelina benghalensis L., Commelina erecta L. e Croton spp.

As Depressões Rasas - "shallow depressions" em contraste com as cacimbas, são apenas brevemente inundadas, por um a dois dias, após chuvas intensas, no entanto apresentam o acúmulo de uma maior quantidade de substrato. Elas foram responsáveis por abrigar extensas populações, onde predominaram com mais frequência indivíduos de Encholirium spectabile; Mandevilla tenuifolia (J.C. Mikan) Woodson, Marsdenia altissima (Jacq.) Dugand, Marsdenia megalantha Goyder \& Morillo, Peplonia sp.; Cereus jamacaru DC., Pilosocereus gounellei (F.A.C. Weber) Byles \& G.D. Rowley, Pilosocereus chrysostele (Vaupel) Byles \& G.D. Rowley, Tacinga inamoena (K. Schum.) N.P. Taylor \& Stuppy.

As Depressões Profundas - "deep depressions" possibilitam a ocorrência de solos mais desenvolvidos e foram colonizadas por árvores e arbustos de pequeno porte como Aspidosperma pyrifolium Mart., Cochlospermum vitifolium (Willd.) Spreng., Capparis flexuosa (L.), Trema micrantha (L.) Blume, Manihot glaziovii Müll. Arg., Bauhinia pentandra (Bong.) Vogel ex Steud. e Mimosa tenuifolia L..

Os Cinturões Florestados localizam-se no sopé do inselbergue e foram colonizados por árvores de maior porte, que permanecem verdejantes por mais tempo em relação à vegetação circundante. Mimosa tenuifolia Poir e Croton sp. foram dominantes na vegetação da base do inselbergue que historicamente é explorada para a retirada de madeira, plantio e pastoreio.

As Cavernas foram o micro-habitat adicionado na nossa classificação para descrever áreas frequentemente sombreadas, onde foram coletadas cinco espécies herbáceas Philodendron acutatum Schott, Spathicarpa sagittifolia Schott ), Taccarum peregrinum (Schott) Engl., Adiantum deflectens Mart. e Doryopteris concolor (Langsd. \& Fisch.) Kuhn, e duas arbóreas, Ilex spp.

\section{Formas de crescimento e formas de vida}

Registramos que as espécies arbóreas destacaram-se em relação às outras formas de crescimento (Quadro 1). As árvores ocorreram preferencialmente nas fissuras e cinturões florestados, micro-habitat que segundo Porembski (2007) são detentores de maior quantidade de substrato, maior disponibilidade de água e redução da radiação e das taxas de evaporação em relação aos demais. Entre elas destacaramse: Ficus christianii Carauta, Lithraea sp., Cordia oncocalyx Allemão, Anadenanthera colubrina (Vell.) Brenan e Crataeva tapia L.. No entanto, indivíduos jovens de espécies arbóreas raramente foram encontrados, o que pode estar associado à competição, pois nas fissuras o espaço para colonização é restrito. Noort et al. (2007) registraram a presença de Ficus (Moraceae) no monte Namuli (África), e inferiram que as populações são remanescentes de períodos mais úmidos. Além disso, Aspidosperma pyrifolium Mart., Cordia oncocalyx (Allemão) Baill. Encholirium spectabile Mart. ex Schult., Cereus jamacaru, Tacinga inamoena Britton \& Rose, Pilosocereus gounellei (F.A.C. Weber) Byles \& G.D. Rowley, Chloroleucum domosum (Benth.) G.P. Lewis e Ziziphus joazeiro Mart., são espécies endêmicas da caatinga (Giulietti et al. 2004) que também colonizaram a Pedra Derretida e a Pedra do Padre em Quixadá, confirmando as informações de Porembski (2007), Scarano (2007) e Araújo et al. (2008) de que a vegetação circundante é uma importante fonte de propágulos para os afloramentos rochosos.

Quanto às formas de vida, registramos 64 fanerófitos, 25 terófitos, sete caméfitos, seis hemicriptófitos, e cinco geófitos (Quadro 1). Observações de campo permitiram destacar que os geófitos foram às formas de vida mais encontradas com flores no início do período chuvoso, por estarem latentes e com corpo subterrâneo já formado; diferente dos terófitos que ainda teriam que desenvolver um corpo para crescimento de ramos férteis, no entanto, a partir do meio da estação chuvosa, as ervas terofíticas foram as mais encontradas. Araújo et al. (2008) registraram predomínio de terófitos com 34 espécies enquanto os fanerófitos foram representados por apenas 19 espécies. Nossos resultados contrariam esse predomínio de terófitos como a forma de vida dominante nos afloramentos rochosos. Segundo França et al. (2005) diferenças na proporção entre as formas de vida podem estar relacionada à formação do solo e a disponibilidade de água em cada microssítio e/ ou ser decorrente de diferenças na vegetação circundante. Como o inselbergue estudado por Araújo et al. (2008) apresenta a mesma vegetação circundante (Caatinga) dos dois inventariados neste estudo, acreditamos que o predomínio de fanerófito deve-se a peculiaridades de variáveis abióticas locais, como maior disponibilidade de solo e de água em determinados micro-habitat, como fendas, cavernas, cacimbas, depressões e cinturões florestados.

\section{Síndrome de dispersão}

Catalogamos síndromes de dispersão zoocórica em 43 espécies, autocórica em 34 e anemocórica em 30 . Por um lado estes resultados confirmam o padrão esperado para inselbergues de climas secos e úmidos, em que tem sido registrado um predomínio de síndromes abióticas (Barthlott et al. 1993, Ibisch et al. 1995, Porembski et al. 1997, Araújo et al. 2008). Mas, por outro lado, o percentual de zoocoria (40\%) deste estudo foi superior aos $16 \%$ registrados por Araújo et al. (2008) em outro inselbergues Quixadaense.

A maior parte das espécies zoocóricas foi representada por Boraginaceae, Myrtaceae, Moraceae e Salicaceae, e todas foram registradas em micro-habitats sombreados e mais úmidos, como os canais de drenagem, depressões profundas e cinturões florestados (Quadro 1). Além disso, a presença de árvores com frutos drupáceos na borda das cavernas evidencia dispersão zoocórica dos diásporos, e pode ser atribuído não somente a pássaros, mas a morcegos presentes nestas cavernas. Já as espécies anemocóricas estavam diretamente relacionadas a locais expostos ao sol e a micro-habitats mais hostis, como depressão rasa e paredão rochoso, por exemplo, e dentre elas destacam- 
se todos os espécimes de Apocynaceae, Bromeliaceae Orchidaceae.

Em relação à conservação podemos destacar que Trema micranta e Commelina bengalensis são descritas por Lorenzi (2008) como ruderais típicas e foram encontradas em diversos micro-habitats nos inselbergues aqui estudados. Esses dados revelam que apesar do elevado número de endemismos (Porembski 2007) nos inselbergues também ocorrem espécies generalistas, corroborando França et al. (1997). Além disso, foram registradas a ocorrência das invasoras Calotropis procera e Cryptostegia madagascariensis, na "Pedra derretida" (inselbergue com entorno mais devastado). Segundo Gomes \& Alves (2009), espécies ruderais são possivelmente oriundas da região circundante, onde predominam agricultura e pastagem. Esse dado é preocupante, pois de acordo com Silva et al. (2016): i) tanto os inselbergues como as áreas de entorno são constantemente alvos de intensas ações antrópicas, que incluem extração mineral nos afloramentos e substituição da vegetação nativa por agricultura, no entorno e ii) apesar da Caatinga ser o domínio fitogeográfico brasileiro com maior abundância de afloramentos rochosos, o conhecimento sobre a flora desses ecossistemas ainda é incipiente, especialmente, no que concerne à conservação.

\section{CONCLUSÕES}

Concluímos que o predomínio de espécies zoocóricas, fanerófitas e arbóreas, diferente do já registrado para outros inselbergues de regiões semiáridas, se deve principalmente às características intrínsecas dos nove micro-habitat reconhecidos, como quantidade de substrato e disponibilidade hídrica. Uma vez que de acordo com a revisão de Silva (2016), os inselbergues são caracterizados por filtros abióticos de disponibilidade hídrica e temperatura que selecionam espécies diferentes, ainda que equivalentes funcionalmente.

Além disso, a riqueza e a composição de espécies foram maiores e diferiram de outros inselbergues também situados no nordeste semiárido brasileiro. Nossos resultados confirmam a ideia de que o isolamento dos inselbergues funciona como barreira geográfica para grupos vegetais extremamente dependentes de microclimas, colocando-os em uma condição ecológica diferenciada (França et al. 2005 e Silva 2016), subsídio relevante para a conservação integral dessas áreas. Aliado a isso o registro de espécies invasoras é preocupante e reforça a necessidade de manejo e conservação desses ecossistemas e seu entorno.

\section{REFERÊNCIAS}

Almeida, A., Felix, W.P., Andrade, L.A. \& Felix, L.P. 2008. A família Orchidaceae em inselbergues da Paraíba, Nordeste do Brasil. Revista Brasileira de Biociências 5(2):753-755.

Angiosperm Phylogeny Group - APG IV. 2016. An udpdate of the Angiosperm Phylogeny Group classification for the orders and families of flowering plants: APG IV. Botanical Journal of the Linnean Society 85(4):531-553.
Associação Internacional das Montanhas Famosas - WFMA. 2015. Disponível em http://www.s.wfmainbrazil.com/index.php/montanhasno-brasil\#axzz3v4C2d614 Acessado em 22.12.2015.

Araújo, F.S., Oliveira, R.F. \& Lima-Verde, L.W. 2008. Composição, espectro biológico e síndromes de dispersão da vegetação de um Inselbergue no domínio da Caatinga, Ceará. Rodriguésia 59(4):659671.

Araújo, F.S., Rodal, M.J.N., Barbosa, M.R.V. \& Martins, F.R. 2005. Vegetação e flora fanerogâmica da área Reserva Serra das Almas, Ceará. In Análise das variações da Biodiversidade do Bioma Caatinga suporte a estratégias regionais de conservação (F.S. Araújo, M.J.N. Rodal \& M.R.V. Barbosa, orgs.). Ministério do Meio Ambiente, Brasília, p. 94-121.

Barthlott, W., Gröger, A. \& Porembski, S. 1993. Some remarks on the vegetation of tropical inselbergs: diversity and ecological differentiation. Biogeographica 69(3):105-124.

Burke, A. 2002. Island-matrix relationships in Nama Karoo inselberg landscapes Part II: Are some inselbergs better sources than others? Plant Ecology 158(1):41-48.

Burke, A. 2004. From plains to inselbergs: species in special habitats as indicators for climate change?. Journal of Biogeography 31(5):831841.

Caiafa, A.N. \& Silva, A.F. 2007. Structural analysis of the vegetation on a highland granitic rock outcrop in Southeast Brazil. Brazilian Journal of Botany 30(4):657-664.

Carmo, F.F. \& Jacobi, C.M. 2013. A vegetação de canga no Quadrilátero Ferrífero, Minas Gerais: caracterização e contexto fitogeográfico. Rodriguésia 64(3):527-541.

Costa, R.C., Araújo, F.S. \& Lima-Verde, L.W. 2007. Flora and life-form spectrum in an area of deciduous thorn woodland (caatinga) in northeastern, Brazil. Journal of Arid Environments 68(2):237-247.

França, F., Melo, E. \& Santos, C.C. 1997. Flora de inselbergs da região de Milagres Bahia, Brasil: I. Caracterização da vegetação e lista de espécies de dois inselbergs. Sitientibus 17(2):63-184.

França, F., Melo, E., Santos, A.K.A., Melo, J.G.A.N., Marques, M., SilvaFilho, M.F.B. \& Machado, C. 2005. Estudo ecológico e florístico em ilhas de vegetação de um inselberg no semi-árido da Bahia, Brasil. Hoehnea 32(1):93-101.

Fundação Cearense de Meteorologia e Recursos Hídricos - FUNCEME. 2015. Dados dos postos pluviométricos do Ceará. Disponível em: http://www.funceme.br/app/calendario/produto/municipios/maxima/ diario?data $=$ hoje Acessado em 15/10/2015.

Giulietti, A. M., Bocage Neta, A.L., Castro, A.A.J.F., Gamarra-Rojas, C.F.L., Sampaio, E.V.S.B., Virgínio, J.F. \& Harley, R.M. 2004. Diagnóstico da vegetação nativa do bioma Caatinga. In Biodiversidade da Caatinga: áreas e ações prioritárias para a conservação (J.D. Silva, M. Tabarelli, M.D. Fonseca \& L.V. Lins, orgs.) Ministério do Meio Ambiente, Brasília, p. 48-90.

Gomes, P. \& Alves, M. 2009. Floristic and vegetational aspects of an inselberg in the Semi-Arid region of Northeast Brazil. Edinburgh Journal of Botany 66(2):329-346.

Griz, L.M.S. \& Machado, I.C. 2001. Fruiting phenology and seed dispersal syndromes in caatinga, a tropical dry forest in the northeast Brazil. Journal of Tropical Ecology 17(2):303-321.

Gröger, A. \& Huber, O. 2007. Rock outcrop habitats in the Venezuelan Guayana lowlans: their main vegetation types and floristic componentes. Revista Brasileira de Botânica 30(4):599-609.

Ibisch, P.L., Rauer, G., Rudolph, D. \& Barthlott, W. 1995. Floristic, biogeographical, and vegetational aspects of pre-cambrian rocks outcrops (inselbergs) in eastern Bolivia. Flora 190(1):299-314.

Instituto Nacional de Meteorologia - INMET. 2015. Banco de Dados Meteorológicos para Ensino e Pesquisa. Disponível em: http://www. inmet.gov.br/portal/index.php?r=bdmep/bdmep.

International Plant Names Index - IPNI. 2015. Published on the Internet. Disponível em: $<\mathrm{http}: / /$ www.ipni.org $>$.

Jardim Botânico do Rio De Janeiro - JBRJ. 2016. Flora do Brasil 2020 em construção. Rio de Janeiro: Jardim Botânico do Rio de Janeiro. Disponível em: <http://floradobrasil.jbrj.gov.br $>$.

Lorenzi, H. 2008. Plantas daninhas do Brasil: terrestres, aquáticas, parasitas e tóxicas. Instituto Plantarum de Estudos da Flora, São Paulo. $672 \mathrm{p}$. 
Lumaret, R., Guillerm, J., Maillet, J. \& Verlaque, R. 1997. Plant species diversity and polyploidy in islands of natural vegetation isolated in extensive cultivated lands. Biodiversity and Conservation 6(4):591-613.

MaCarthur, R.H. \& Wilson, E.O. 1967. The Theory of Island Biogeography. Princeton University Press, Princeton. 224 p.

Martinelli, G. \& Forzza, R.C. 2006. Pitcairnia L'Hér.(Bromeliaceae): a new species, $P$. azouryi Martinelli \& Forzza, and notes on $P$. encholirioides LB Sm. Brazilian Journal of Botany 29(4):603-607.

Martins, S. \& Alves, M. 2008. Aspectos anatômicos de espécies simpátridas de Mandevilla (Apocynaceae) ocorrentes em três inselbergues de Pernambuco-Brasil. Rodriguésia 59(2):369-380.

Medina, B.M.O., Ribeiro, K.T. \& Scarano, F.R. 2006. Plant-plant and plant-topography interactions on a rock outcrop at high altitude in southeastern Brazil. Biotrópica 38(1):27-34.

Meirelles, S.T., Pivello, V.R. \& Joly, C.A. 1999. The vegetation of granite rock outcrops in Rio de Janeiro, Brazil, and the need for its protection. Environmental Conservation 26(1):10-20.

Menezes, M.O.T., Araújo, F.S. \& Romero, R.E. 2010. O sistema de conservação biológica do Estado do Ceará: diagnóstico e recomendações. Rede 5(2):7-31.

Moraes, A.O., Melo, E.G, Agra, M.F. \& França, F. 2009. A família Solanaceae nos inselbergues do semi-árido da Bahia, Brasil. Iheringia. Série Botânica 64(2):109-122.

Noort, S.V., Gardiner, A.J. \& Tolley, K.A. 2007. New records of Ficus (Moraceae) species emphasize the conservation significance of inselbergs in Mozambique, South African. Journal of Botany 73(4):642-649.

Parmentier, I. 2003. Study of the vegetation composition in three inselbergs from continental Equatorial Guinea (Western Central Africa): Efects of site, Soil factors and position relative to forest fringe. Belgian Journal of Botany 136(1):63-72.

Parmentier, I., Stévart, T. \& Hardy, O. J. 2005. The inselberg flora of Atlantic Central Africa. I. Determinants of species. Journal of Biogeography 32(4):685-696.
Porembski, S. 2007. Tropical inselbergs: habitat types, adaptive strategies and diversity patterns. Brazilian Journal of Botany 30(4):579-586.

Porembski, S. \& Barthlott, W. 2000. Granitic and gneissic outcrops (inselbergs) as centers of desiccation-tolerant vascular plants. Plant Ecology 151(1):19-28.

Porembski, S., Martinelli, G., Ohlemüller, R. \& Barthlott, W. 1998. Diversity and ecology of saxicolous vegetation mats on inselbergs in the Brazilian Atlantic rainforest. Diversity and distributions 4(3):107-119.

Porembski, S., Seine, R. \& Barthlott, W. 1997. Inselberg vegetation and biodiversity of granite outcrops. Journal of the Royal Society of Western Australia 80(3):193-199.

Porto, P.A.F., Almeida, A., Pessoa, W.J., Felix, L.P. \& Trovão, D. 2008. Composição florística de um inselbergue no Agreste paraibano, município de Esperança, Nordeste do Brasil. Revista Caatinga 21(2):214-223

Ratter, J.A., Bridgewater, S., Atkinson, R. \& Ribeiro, J.F. 1996. Analysis of the floristic composition of the Brazilian cerrado vegetation II: comparison of the woody vegetation of 98 areas. Edinburgh Journal of Botany 53(2):153-180.

Raunkiaer, C. 1934. The life forms of plants and statistical plant geography. Clarendon Press, Oxford. 632 p.

Ribeiro, S.C., Marçal, M.S. \& Correa, A.C.B. 2010. Geomorfologia de áreas semi-áridas: uma contribuição ao estudo dos sertões nordestinos. Revista de Geografia da Universidade Federal de Pernambuco 27(1):120-137.

Silva, J.B. 2016. Panorama sobre a vegetação em afloramentos rochosos do Brasil. Oecologia Australis 20(4):451-463.

Sarthou, C., Kounda-Kiki, C., Vaçulik, A., Mora, P., \& Ponge, J. F. (2009). Successional patterns on tropical inselbergs: A case study on the Nouragues inselberg (French Guiana). Flora-Morphology, Distribution, Functional Ecology of Plants 204(5):396-407.

Scarano, F.R. 2007. Rock outcrop vegetation in Brazil: a brief overview. Brazilian Journal of Botany 30(4):561-568.

Van der Pijl, L. 1982. Principles of dispersal in higher plants. Springer Verlag, Berlin. 215p. 